\title{
Safety and Efficacy of Thrombolysis in Cervical Artery Dissection-Related Ischemic Stroke: A Meta-Analysis of Observational Studies
}

\author{
Jueying Lin ${ }^{a} d \quad$ Yefei Sun $^{b}$ Shanshan Zhao ${ }^{a} \quad J u n j i e ~ X u^{c} \quad$ Chuansheng Zhao $^{a}$ \\ ${ }^{a}$ Department of Neurology, ${ }^{b}$ Gastrointestinal Surgery, and ${ }^{\mathrm{c} D e p a r t m e n t}$ of Laboratory Medicine, The First Hospital \\ of China Medical University, Shenyang, and ' Emergency Department, Zhongshan Hospital Xiamen University, \\ Xiamen, China
}

\section{Key Words}

Cervical artery dissection - Ischemic stroke - Thrombolysis .

Safety · Efficacy · Meta-analysis

\begin{abstract}
Background: Although thrombolysis is considered to be the first-line treatment for ischemic stroke, there remains an ongoing controversy on the safety and efficacy of thrombolysis in cervical artery dissection (CAD). The aim of this meta-analysis was to assess observational data related to the safety and efficacy of thrombolysis in CAD-related ischemic stroke. Methods: We performed a systematic search of the efficacy of thrombolysis treatment in CAD-related ischemic stroke with appropriate observational studies identified for the study. The meta-analysis models in Comprehensive MetaAnalysis V2 software were applied to calculate the merged rates of favorable outcome (modified Rankin Scale, mRS 0-2), excellent outcome (mRS 0-1), intracranial hemorrhage (ICH), symptomatic ICH (SICH), mortality and recurrent stroke between thrombolysis and non-thrombolysis in CAD-related stroke. The difference of outcomes and adverse events between the 2 groups was compared by analyzing the pooled OR value and chi-square test using the software SPSS. Results: A total of 846 patients were identified from 10 studies
\end{abstract}

(174 with thrombolysis; 672 with non-thrombolysis). The meta-analysis detected no significant statistical difference in the proportion of CAD-related stroke patients enjoying a favorable outcome at the 3 months' follow-up between the thrombolysis and non-thrombolysis groups (53.7 vs. $58.2 \%$, OR $0.782, x^{2}=0.594, p>0.05$ ); non-thrombolysis was slightly superior than thrombolysis in terms of excellent outcome ( 52.4 vs. $34.4 \%$, OR $0.489, x^{2}=9.143, p=0.002$ ). There was no significant difference in $\mathrm{SICH}$, mortality and recurrent stroke rates between the 2 groups (all $p>0.05$ ). ICH rate was higher in the thrombolysis group of CAD-related stroke patients compared to that in the non-thrombolysis group (12.3 vs. $7.4 \%$, OR 2.647, $x^{2}=4.127, p=0.042$ ). Conclusion: Thrombolysis seems to be equally safe and will achieve an efficacy similar to the efficacy of non-thrombolysis in patients with acute ischemic stroke due to CAD. It is also as effective as thrombolysis in stroke from miscellaneous causes. Therefore, CAD patients experiencing a stroke should not be denied thrombolysis therapy. However, this will need to be confirmed in large-scale randomized studies, especially involving intravenous thrombolysis treatment.

(c) 2016 S. Karger AG, Basel

Lin J.Y. and Sun Y.F. contributed to this work equally.

\section{KARGER}

E-Mail karger@karger.com

www.karger.com/ced
(C) 2016 S. Karger AG, Basel

$1015-9770 / 16 / 0424-0272 \$ 39.50 / 0$
Chuansheng Zhao

Department of Neurology

The First Hospital of China Medical University

No. 155, North Nanjing Street, Heping District, Shenyang, Liaoning 110001 (China)

E-Mail cszhao@mail.cmu.edu.cn 


\section{Introduction}

The incidence of stroke has been on the rise in recent years, especially stroke in younger individuals. Cervical artery dissection (CAD) can lead to occlusion of the artery [1] and it has been estimated that $2 \%$ of all ischemic strokes are caused by CAD. In fact, CAD is a major cause in stroke of young people [2], for example, in patients under the age of 50, CAD is a rather common cause of stroke, accounting for $10-25 \%$ of all ischemic strokes [3-5]. At present, many studies have indicated that thrombolysis may improve the prognosis by restoring perfusion of cerebral blood flow; it is safe and effective in acute ischemic stroke $[6,7]$. In fact, the guideline for CAD does not exclude thrombolysis [8], and randomized controlled trials that have investigated thrombolysis in acute ischemic have not excluded patients with CAD [9]. On the other hand, CAD consists of internal carotid artery dissection and vertebral artery dissection, which is caused by the presence of disturbed hemodynamics and abnormalities in the structure of arterial wall [10-12]. In theory, there is a danger that thrombolysis may increase the risk of enlargement of an intramural hematoma in the dissected artery and cause local complications or further impair the cerebral circulation [13]. As the pathogenesis of CAD is associated with a high risk of thrombolysis, the safety and efficacy of thrombolysis in this group of patients have been a matter of intense debate. The aim of this metaanalysis was to assess the safety and efficacy of thrombolysis in CAD-related ischemic stroke by evaluating all available observational studies.

\section{Methods}

\section{Search Strategy}

We systematically searched PubMed, Web of Science, EMBASE, Cochrane Library, CNKI database up to May 2015. We used the following combination of variables: ('carotid artery dissection' or 'vertebral artery dissection' or 'cervical artery dissection') and ('stroke' or 'brain ischemia' or 'brain infarction') and ('thrombolysis' or 'recombinant tissue plasminogen activator' or 'rtPA' or 'tissue plasminogen activator' or 'tPA' or 'urokinase' or 'pro-urokinase'). The study languages were restricted to English and Chinese. In addition, the reference lists of each obtained article were manually examined to identify any additional potentially relevant citations.

\section{Selection Criteria and Quality Assessment}

Articles were considered eligible if they met the following criteria: (1) investigation of thrombolysis and non-thrombolysis or only thrombolysis in patients with stroke due to CAD, either as the main focus of the research or as a subgroup analysis. (2) All patients who had CAD-related ischemic stroke and ischemic stroke had been diagnosed according to the WHO criteria [14]. (3) The diagnosis of CAD needed to be confirmed with the appropriate imaging tests: color duplex sonography, CT angiography, magnetic resonance angiography, or digital subtraction angiography. (4) Observational studies. (5) The loss rate was less than $10 \%$.

Patients with an intracranial extension of dissection or with some other artery dissection extending into the cervical arteries were excluded. In addition, we also excluded individual case reports. Since there are only a few studies that have investigated on this topic, we decided to include the criterion that there should be a minimum number of 3 patients evaluated in the study.

Two authors reviewed the articles to judge their eligibility independently and evaluated the articles according to the standard checklist of Strengthening the Reporting of Observational Studies in Epidemiology (STROBE), with disagreements settled by discussion between the authors until a consensus was reached. The STROBE checklist was designed and used in the current systematic review; it includes several questions about methodological aspects of the report, such as type of study, study objectives, characteristics of the study population, clearly explained inclusion/exclusion criteria, data collection method and instrument, variable definition, bias, study size, and statistical tests $[15,16]$. Non-qualified studies were excluded.

\section{Data Extraction}

We analyzed 2 different types of treatment groups in thrombolysis: intravenous thrombolysis (IVT) and other thrombolytic treatment, the latter being defined as treatment with intra-arterial thrombolysis (IAT) only or IVT followed by IAT. Both authors extracted data from each pooled study independently. We collated the following baseline characteristics: year, country, treatment type (thrombolysis or non-thrombolysis treatment), patient number, stroke severity (NIHSS score), proportion of favorable functional outcome (modified Rankin Scale, mRS 0-2), proportion of excellent functional outcome (mRS 0-1), proportion of intracranial hemorrhage $(\mathrm{ICH})$, proportion of symptomatic $\mathrm{ICH}(\mathrm{SICH})$, proportion of mortality, proportion of recurrent stroke and follow-up duration.

\section{Statistical Methods}

Meta-analysis was carried out using the Comprehensive MetaAnalysis V2 (CMA V2) software. We calculated the merged rate and its $95 \%$ CI in the analysis. The heterogeneity of the studies was assessed using $\mathrm{I}^{2}$. A value of $\mathrm{I}^{2}<50 \%$ indicated homogeny and the fixed effects model was used in the analysis. In contrast, if $\mathrm{I}^{2}>50 \%$, this was interpreted as representing significant heterogeneity and we used a random effects model to assess differences and subgroup analyses were implemented. Funnel plot, Begg's test and Egger's test were adopted to test the possible publication bias $(\mathrm{p}>0.05$, no significant publication bias). We merged the rate of outcome and adverse event both in pooled thrombolysis and non-thrombolysis group, and the pooled OR value ( $95 \% \mathrm{CI}$ ) and chi-square test were calculated by SPSS software to compare the difference between the 2 groups.

\section{Results}

\section{Literature Search}

Our search strategy retrieved 215 results (study selection flow diagram is shown in figure 1), but only 10 stud- 
ies fulfilled our predefined criteria [4, 17-25], including 6 studies without a control group (patients with thrombolysis treatment only in CAD-related stroke) and 4 studies with both thrombolysis and non-thrombolysis groups. All studies were observational studies (retrospective studies); no randomized controlled trials were identified. Three of the studies had been conducted as multicenter trials [19, 20,24]. In total, 846 patients were available for our analysis, including 174 thrombolysis patients; of these, 148 had been treated with IVT and the remaining 26 had been administered some other form of thrombolytic treatment. There were 672 non-thrombolysis patients.

\section{Baseline Characteristics of the Eligible Studies}

The baseline characteristics of the 10 eligible studies are shown in table 1; all of the investigated patients were of European origin. First, patients with CAD-related stroke from 10 studies were treated for thrombolysis; however, only 4 research reports from these studies were included for non-thrombolysis treatment. We found that there were no significant differences between the 10 studies in terms of their patients' age, gender, location of dissection artery, and vascular risk factors of patients such as hypertension, diabetes mellitus, hypercholesterolemia and smoking status. However, the NIHSS score at presentation was clearly different; for instance, the non-thrombolysis group in the report of Fuentes et al. [19] and Engelter et al. [20] had a mild stroke severity at presentation, whereas the thrombolysis group investigated in the reports of Arnold et al. [21] and Rudolf et al. [23] had more severe strokes at presentation. As the outcome would be influenced by the different stroke severities at presentation, we obtained data adjustment for NIHSS score from the original literature (Engelter et al. [20], Fuentes et al. [19], Arnold et al. [21] and Rudolf et al. [23]).

\section{Result of the Meta-Analysis}

We assessed the prognosis of ischemic stroke in terms of the functional outcome at the 3 months' follow-up. The mRS score was used to evaluate the efficacy in these 10 studies, including favorable functional outcome and excellent functional outcome. A favorable outcome was defined as an mRS score of $0-2$; the excellent outcome was in the range $0-1$. We analyzed several safety variables, that is, $\mathrm{ICH}, \mathrm{SICH}$, mortality, and recurrent stroke. We used the same definition of SICH as in SITS-MOST [26]: a local or remote type 2 parenchymal hemorrhage on imaging 22-36 h after treatment, combined with

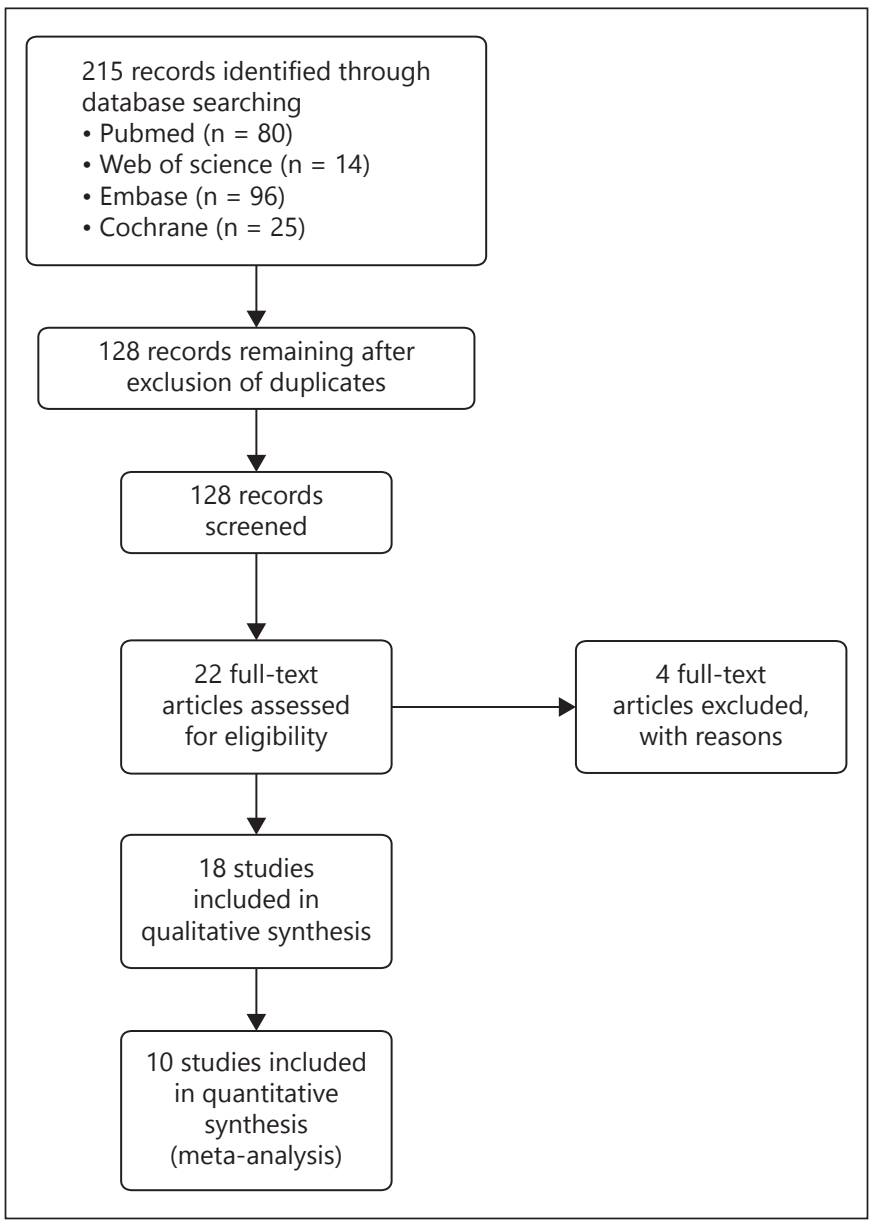

Fig. 1. Flow diagram for identification of relevant studies.

a neurological deterioration of 4 or more points on the NIHSS from baseline or from the lowest NIHSS score between baseline and $24 \mathrm{~h}$, or a hemorrhage leading to death.

In terms of efficacy, we analyzed the merged rates of favorable functional outcome and excellent functional outcome between the thrombolysis and the non-thrombolysis groups from the examined studies. Significant heterogeneity was present only in the excellent functional outcome of the non-thrombolysis group $\left(\mathrm{I}^{2}=\right.$ $79.266, \mathrm{p}=0.002$ ) and no significant heterogeneity was observed between any of the other groups $\left(\mathrm{I}^{2}<50 \%\right.$, p > $0.05)$. Thus, a random effects model was used to evaluate the excellent functional outcome in the non-thrombolysis group; other groups were examined with a fixed effects model. The meta-analysis revealed that the merged rates of favorable functional outcome in thrombolysis and non-thrombolysis groups were respectively 53.7\% (95\% CI 45.9-61.3) and 58.2\% (95\% CI 47.2-68.4; 
table 2). No significant differences in favorable functional outcome at 3 months were found when comparing the thrombolysis group with their non-thrombolysis counterparts (pooled OR 0.782, 95\% CI 0.495-1.332, $\chi^{2}=0.594, \mathrm{p}>0.05$; table 3 ). Furthermore, the merged rates of excellent functional outcome were $34.4 \%$ (95\% CI 27.0-42.7) in the thrombolysis group and 52.4\% (95\% CI 32.8-71.3) in the non-thrombolysis group (table 2). There was a significant difference in excellent functional outcome at 3 months between the 2 groups (pooled OR 0.489, 95\% CI 0.312-0.767, $\chi^{2}=9.143$, p < 0.05 ; table 3 ). A similar result was obtained when we assessed the number of CAD-related stroke patients enjoying a favorable outcome at the 3 months' follow-up between the 2 groups; non-thrombolysis was slightly superior over thrombolysis in terms of likelihood of achieving an excellent outcome.

We analyzed the merged rates of several safety variables, that is, $\mathrm{ICH}, \mathrm{SICH}$, mortality, and recurrent stroke between the thrombolysis and the non-thrombolysis groups from the examined studies. Only one patient experienced an SICH after non-thrombolysis treatment; there were no incidents of SICH in the thrombolysis group. Because of the low incidence of SICH, we could not calculate the merged rates of SICH in both the groups with the CMA software. When the different research reports were evaluated, significant heterogeneity was present only in ICH in the non-thrombolysis group $\left(\mathrm{I}^{2}=85.563, \mathrm{p}=0.000\right)$; no significant heterogeneity was observed in the other safety variables $\left(\mathrm{I}^{2}<50 \%, \mathrm{p}>0.05\right)$. A random effects model was used to assess the possibility of ICH in the non-thrombolysis group, while the fixed effects model was adopted for the other groups. The meta-analysis showed that the ICH merged rate in the thrombolysis group was $12.3 \%$ (95\% CI 7.5-19.4), whereas in the non-thrombolysis group, it was $7.4 \%$ (95\% CI 0.6-51.3); the merged rates of mortality were $7.8 \%$ (95\% CI 3.8-15.3) in the thrombolysis group and $1.6 \%$ (95\% CI $0.5-5.5)$ in the non-thrombolysis group; the recurrent stroke merged rate in the thrombolysis group was $4.9 \%$ (95\% CI 2.0-11.2) and in the nonthrombolysis group, it was $6.3 \%$ (95\% CI 3.3-11.9; table 2). No significant differences in mortality and recurrent stroke were found when comparing the thrombolysis group with their non-thrombolysis counterparts (pooled OR 6.361, 95\% CI 0.735-55.038, $\chi^{2}=2.268$, p > 0.05 ; pooled OR $0.522,95 \%$ CI $0.135-2.011, \chi^{2}=0.420$, $\mathrm{p}>0.05$; table 3 ). In addition, as no SICH occurred in the thrombolysis group, we found that thrombolysis was not associated with $\mathrm{SICH}$, mortality and recurrent

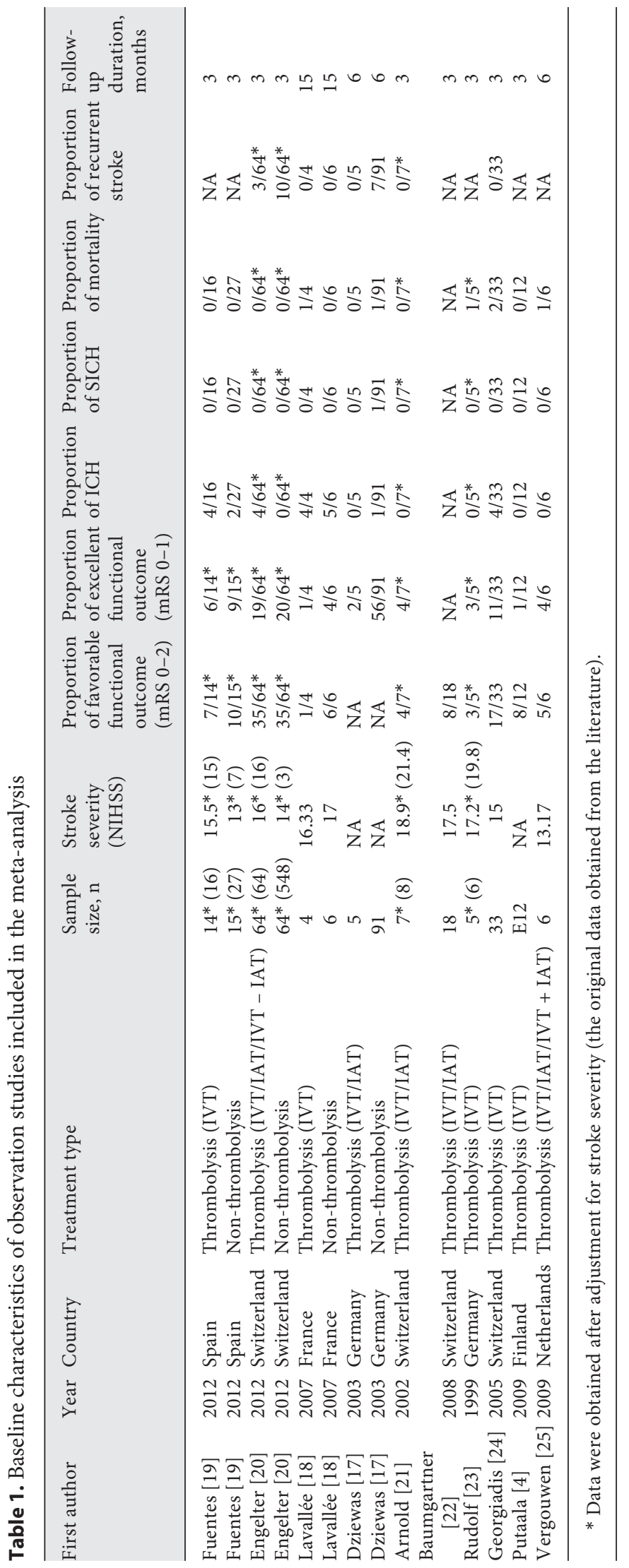

Cerebrovasc Dis 2016;42:272-279

DOI: $10.1159 / 000446004$
Safety and Efficacy of Thrombolysis in

CAD-Related Ischemic Stroke 
Table 2. Meta-analysis of event merged rates between the thrombolysis group and the non-thrombolysis group in CAD-related stroke

\begin{tabular}{|c|c|c|c|c|c|c|c|}
\hline Events & Thrombolysis type & $\begin{array}{l}\text { Number of } \\
\text { studies }\end{array}$ & $\begin{array}{l}\text { Heterogeneity, } \\
\mathrm{I}^{2} \text { value }\end{array}$ & $\mathrm{p}$ value & $\begin{array}{l}\text { Effected } \\
\text { models of } \\
\text { meta-analysis }\end{array}$ & \multicolumn{2}{|c|}{ Result of meta-analysis } \\
\hline \multirow{2}{*}{$\begin{array}{l}\text { Favorable functional } \\
\text { outcome (mRS 0-2) }\end{array}$} & Thrombolysis & 9 & 0.000 & 0.795 & Fixed & $53.7(45.9-61.3)$ & 0.351 \\
\hline & Non-thrombolysis & 3 & 35.028 & 0.215 & Fixed & $58.2(47.2-68.4)$ & 0.144 \\
\hline \multirow[t]{2}{*}{$\mathrm{ICH}$} & Thrombolysis & 9 & 40.621 & 0.097 & Fixed & $12.3(7.5-19.4)$ & 0.000 \\
\hline & Non-thrombolysis & 4 & 85.563 & 0.000 & Random & $7.4(0.6-53.1)$ & 0.062 \\
\hline \multirow[t]{2}{*}{ Mortality } & Thrombolysis & 9 & 0.000 & 0.654 & Fixed & $7.8(3.8-15.3)$ & 0.000 \\
\hline & Non-thrombolysis & 4 & 0.000 & 0.672 & Fixed & $1.6(0.5-5.5)$ & 0.000 \\
\hline
\end{tabular}

Table 3. The pooled OR and 95\% CI in adverse events and outcome between the thrombolysis group and the non-thrombolysis group in CAD-related stroke

\begin{tabular}{|c|c|c|c|c|c|}
\hline \multirow[t]{2}{*}{ Events } & \multicolumn{2}{|l|}{ Proportion, \% } & \multirow[t]{2}{*}{ Pooled OR (95\% CI) } & \multicolumn{2}{|c|}{ Chi-square test } \\
\hline & $\begin{array}{l}\text { thrombolysis } \\
\text { treatment }\end{array}$ & $\begin{array}{l}\text { non-thrombolysis } \\
\text { treatment }\end{array}$ & & $\chi^{2}$ & $\mathrm{p}$ value \\
\hline Favorable functional outcome & $54.0(88 / 163)$ & $60.0(51 / 85)$ & $0.782(0.495-1.332)$ & 0.594 & 0.441 \\
\hline Excellent functional outcome & $33.3(50 / 150)$ & $50.6(89 / 176)$ & $0.489(0.312-0.767)$ & 9.143 & 0.002 \\
\hline $\mathrm{ICH}$ & $10.5(16 / 152)$ & $4.3(8 / 188)$ & $2.647(1.101-6.365)$ & 4.127 & 0.042 \\
\hline $\mathrm{SICH}$ & $0.0(0 / 152)$ & $0.5(1 / 188)$ & $0.615(0.020-18.461)$ & $5.6521 \mathrm{e}-31^{\#}$ & $1.000^{\#}$ \\
\hline Mortality & $3.3(5 / 152)$ & $0.5(1 / 188)$ & $6.361(0.735-55.038)$ & 2.268 & 0.132 \\
\hline Recurrent stroke & $2.7(3 / 113)$ & $5.0(8 / 161)$ & $0.522(0.135-2.011)$ & 0.420 & 0.517 \\
\hline
\end{tabular}

\# As no SICH occurred in the thrombolysis group and only 1 patient experienced this side effect in the non-thrombolysis group, the count of pooled OR and chi-square test must be viewed with caution.

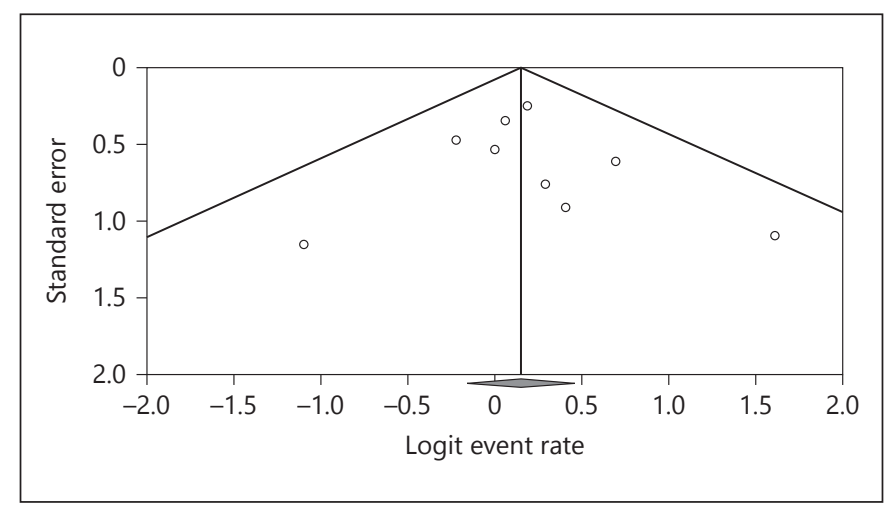

Fig. 2. Funnel plots of standard error by logit event rate. stroke. However, there was a significant difference in $\mathrm{ICH}$ between the 2 groups, that is, the $\mathrm{ICH}$ rate was higher in the thrombolysis group of CAD-related stroke patients compared to that in the non-thrombolysis group (pooled OR 2.647, 95\% CI 1.101-6.365, $\chi^{2}=4.127$, $\mathrm{p}<0.05$; table 3 ).

Funnel plots detected no significant asymmetry in the above meta-analysis (one of the funnel plots; fig. 2). The figures of Begg's and Egger's test in table 4 indicated that there was no obvious publication bias in outcomes and adverse events, with the exception of the favorable functional outcome of non-thrombolysis treatment group (Begg's test: $p>0.05$, Egger's test: $p=0.04$ ). The difference 
Table 4. Publication bias in our meta-analysis (Begg's and Egger's test)

\begin{tabular}{|c|c|c|c|c|c|c|}
\hline Events & Thrombolysis type & $\begin{array}{l}\text { Number of } \\
\text { studies }\end{array}$ & Kendall's tau & $\mathrm{p}$ value & $\mathrm{t}$ value & $\mathrm{p}$ value \\
\hline $\begin{array}{l}\text { Favorable functional } \\
\text { outcome (mRS 0-2) }\end{array}$ & Non-thrombolysis & 3 & 1.000 & 0.296 & 16.021 & 0.040 \\
\hline $\begin{array}{l}\text { Excellent functional } \\
\text { outcome (mRS } 0-1)\end{array}$ & Thrombolysis & 9 & -0.086 & 0.835 & 0.641 & 0.542 \\
\hline $\mathrm{ICH}$ & Non-thrombolysis & 4 & 0.000 & 0.734 & 0.102 & 0.928 \\
\hline \multirow[t]{2}{*}{ Mortality } & Thrombolysis & 9 & 0.000 & 0.917 & 0.624 & 0.552 \\
\hline & Non-thrombolysis & 4 & 0.667 & 0.308 & 0.763 & 0.525 \\
\hline Recurrent stroke & Thrombolysis & 5 & 0.800 & 0.086 & 0.247 & 0.821 \\
\hline
\end{tabular}

between the assessments with Begg's and Egger's test might be attributable to the small number of studies investigating non-thrombolysis treatment.

\section{Discussion}

Although thrombolysis seems to be safe and efficacious in patients suffering an acute ischemic stroke [7], concerns have been raised recently about whether thrombolysis is beneficial and/or safe for those patients who have experienced stroke due to CAD. There are no RCTs in thrombolytic therapy in CAD patients for ischemic stroke. This is the first meta-analysis that has evaluated the safety and efficacy of thrombolysis in ischemic stroke related to $\mathrm{CAD}$ by conducting stratified analyses on favorable functional outcome, excellent functional outcome, ICH, SICH, mortality, and recurrent stroke between thrombolysis and non-thrombolysis groups. The overall findings suggested that thrombolysis in acute ischemic stroke due to $\mathrm{CAD}$ was safe, with a low rate of SICH complications and the same mortality and recurrent stroke rate as in the non-thrombolysis group. After adjustment for stroke severity, almost the same opportunity to recover favorably was found in these 2 groups. In addition, we also compared our thrombolysis data with controls from the SITS-ISTR [27], matched according to age and NIHSS score, to assess the safety and efficacy of thrombolysis in CAD-related stroke.

The efficacy of thrombolysis in acute ischemic stroke due to CAD was assessed by comparing patients with non-thrombolysis against those receiving thrombolysis after a stroke caused by any other disease. In the first stage, our meta-analysis showed that although slightly more patients achieved an excellent functional outcome in the non-thrombolysis group, there was really no difference between the groups if one compares the numbers achieving at least a favorable functional outcome. In addition, we found that more patients with IVT (55.2\%) were achieving a favorable functional outcome than patients with other thrombolytic treatment (45.8\%). Furthermore, we compared our thrombolysis group with controls from the SITS-ISTR, matched for age and NIHSS score. The SITS-ISTR data indicated that $37.4 \%$ of patients enjoyed an excellent functional outcome and $52.2 \%$ reached favorable functional outcome; the corresponding values in our thrombolysis data were 34.4 and $53.7 \%$. In other words, there seemed to be similar outcomes, with patients just as likely to experience a favorable outcome or an excellent outcome after 3 months' follow-up between our thrombolysis data and the SITS-ISTR data. Therefore, the efficacy of thrombolysis in patients with ischemic stroke due to CAD is virtually identical to nonthrombolysis treatment, and is similar to the efficacy of this treatment in patients with stroke from all causes. Furthermore, IVT seems to have a higher efficacy compared with other types of thrombolysis treatment in these patients, and in the future, we intend to carry out the RCT tests on IVT therapy in patients who have experienced a CAD-related stroke.

The safety of thrombolysis in acute ischemic stroke due to CAD was evaluated just as its efficacy was assessed. First, we compared the safety aspects of the thrombolysis therapy and non-thrombolysis therapy. In our meta- 
analysis, the ICH rate was increased in the thrombolysis group compared to the non-thrombolysis group. However, a majority of incidents of ICH in the thrombolysis group were asymptomatic $\mathrm{ICH}$, which has a low risk of mortality. In addition, the SICH rate, overall mortality and recurrent stroke rate in thrombolysis were similar to those in patients not receiving thrombolytic drugs. Thus, the risk of death due to hemorrhage was not increased in the thrombolysis group. Subsequently, we compared our data in the thrombolysis group with that of those from the SITS-ISTR group. Mortality in SITS-ISTR was 8.8 and $3.0 \%$ had SICH. In our data, mortality was lower; it was only $7.8 \%$ and not one patient experienced an SICH. This result is evidence of the fact that the risk of SICH and mortality does not seem to be greater than in other patients with stroke from all causes after matching for age and stroke severity. Therefore, provision of thrombolysis to those patients with acute ischemic stroke due to CAD seems to be equally safe as provision of non-thrombolysis with regard to the potential hemorrhage complications. Furthermore, the risk of serious side effects is the same as encountered when patients receive thrombolysis after a stroke caused by any other disease.

The current treatment guidelines for CAD neither recommend thrombolysis nor exclude this treatment [8]. One systematic review proposed that after adjustment for confounding variables, the chances of making a favorable recovery were virtually identical for IVT and non-IVT treated CAD patients [28], in agreement with our result. In addition, a meta-analysis of individual patient data suggested that the outcome in patients with stroke due to CAD or all causes after thrombolysis is in the same range [29].

The pathophysiologic feature of CAD is an intramural hematoma [30], which is thought to pose a high risk if the patient receives thrombolysis treatment. Therefore, the main adverse event associated with this treatment is a hemorrhage such as ICH and SICH, with SICH being the leading cause of death. There is one meta-analysis of retrospective cases in which patients had received thrombolysis after experiencing a CAD-related stroke [29]; this reported that $3.1 \%$ suffered SICH, and in these patients, mortality was $9.0 \%$, which are similar rates as found in our meta-analysis and also in the European Cooperative Acute Stroke Study III studies and SITS-ISTR [27, 31]. In addition, we found that patients with intracranial extension of dissection had a high SICH rate and mortality in a multicenter study [32], in agreement with the view of some clinicians, fearing that sub-adventitial dissections with intracranial extension especially in posterior circula- tion treated with tPA could cause bleeding into the adjacent tissue, resulting in SICH or subarachnoid hemorrhage $[22,33,34]$. However, 3 earlier studies indicated that thrombolysis was not any more likely to adversely affect patients with intracranial extension of dissection more than other patients [35-37]. Therefore, it still needs to be confirmed whether it is important to conduct vascular imaging to exclude the possibility that the pathology extends dissection intracranial artery before the clinician should consider the administration of thrombolysis.

Our study also had some limitations. First, no randomized trials were available for inclusion in this metaanalysis. The pooled observational studies might influence the reliability of our study. Another limitation was that the types of antithrombotic treatment before and after thrombolysis were not assessed, and this might have affected the adverse events such as $\mathrm{ICH}$ rate, $\mathrm{SICH}$ rate as well as the outcome [1]. In addition, there was a relatively small number of published reports providing details of the variables in the non-thrombolysis group. This reduced the power of the statistical analysis after stratified analysis for certain aspects, such as favorable functional outcome. Therefore, it is clear that further meta-analyses will be needed in this topic after the publication of more relevant investigations.

\section{Conclusions}

Thrombolysis seems to be equally safe and is likely to achieve an identical efficacy as non-thrombolysis in patients with acute ischemic stroke due to CAD. There are similar risks of adverse events when thrombolysis is provided to patients with CAD-related stroke (i.e. SICH rate, mortality and recurrent stroke rate) as encountered with non-thrombolysis as well as in patients receiving thrombolysis from all causes after matching for age and stroke severity. Patients with stroke due to CAD in thrombolysis seem to obtain similar benefits compared with those with miscellaneous causes, as well as, no differences in the outcome at 3 months were found when comparing with patients in non-thrombolysis after adjustment stroke severity. Therefore, thrombolysis treatment in stroke should not be precluded in CAD patients. Since the population is not large enough in our study, it is not possible to recommend that thrombolysis should invariably be provided in the treatment of CAD-related stroke; that recommendation can be made only after further large-scale randomized studies are conducted, especially those investigating the benefits and risks of IVT treatment. 


\section{References}

1 Lyrer P, Engelter S: Antithrombotic drugs for carotid artery dissection. Cochrane Database Syst Rev 2010;10:CD000255.

2 Schievink WI: Spontaneous dissection of the carotid and vertebral arteries. N Engl J Med 2001;344:898-906.

3 Putaala J, Metso AJ, Metso TM, et al: Analysis of 1008 consecutive patients aged 15 to 49 with first-ever ischemic stroke: the Helsinki young stroke registry. Stroke 2009;40:1195-1203.

4 Putaala J, Metso TM, Metso AJ, et al: Thrombolysis in young adults with ischemic stroke. Stroke 2009;40:2085-2091.

5 Vergouwen MD: Intravenous thrombolysis in ischaemic stroke secondary to cervical artery dissection: safe but not effective? Eur J Neurol 2012;19:1155-1156.

6 Wardlaw JM, Murray V, Berge E, del Zoppo GJ: Thrombolysis for acute ischaemic stroke. Cochrane Database Syst Rev 2014;7: CD000213.

7 Tissue plasminogen activator for acute ischemic stroke. The National Institute of Neurological Disorders and Stroke rt-PA Stroke Study Group. N Engl J Med 1995;333:15811587.

8 Jauch EC, Saver JL, Adams HP Jr, et al: Guidelines for the early management of patients with acute ischemic stroke: a guideline for healthcare professionals from the American Heart Association/American Stroke Association. Stroke 2013;44:870-947.

9 Wardlaw JM, Murray V, Berge E, et al: Thrombolysis for acute ischaemic stroke. Cochrane Database Syst Rev 2009;4:CD000213.

10 Schievink WI, Wijdicks EF, Kuiper JD: Seasonal pattern of spontaneous cervical artery dissection. J Neurosurg 1998;89:101-103.

11 Lucas C, Moulin T, Deplanque D, et al: Stroke patterns of internal carotid artery dissection in 40 patients. Stroke 1998;29:2646-2648.

12 Benninger DH, Georgiadis D, Kremer C, et al: Mechanism of ischemic infarct in spontaneous carotid dissection. Stroke 2004;35:482485.

13 Intracerebral hemorrhage after intravenous t-PA therapy for ischemic stroke. The NINDS t-PA Stroke Study Group. Stroke 1997;28: 2109-2118.

14 Stroke - 1989. Recommendations on stroke prevention, diagnosis, and therapy. Report of the WHO Task Force on Stroke and Other Cerebrovascular Disorders. Stroke 1989;20: 1407-1431.
15 Vandenbroucke JP, von Elm E, Altman DG, et al: Strengthening the reporting of observational studies in epidemiology (STROBE): explanation and elaboration. Ann Intern Med 2007;147:W163-W194.

16 Von Elm EV, Altman DG, Egger M, et al: [The strengthening the reporting of observational studies in epidemiology (STROBE) statement: guidelines for reporting of observational studies]. Internist (Berl) 2008;49:688-693.

17 Dziewas R, Konrad C, Dräger B, et al: Cervical artery dissection - clinical features, risk factors, therapy and outcome in 126 patients. J Neurol 2003;250:1179-1184.

18 Lavallée PC, Mazighi M, Saint-Maurice JP, et al: Stent-assisted endovascular thrombolysis versus intravenous thrombolysis in internal carotid artery dissection with tandem internal carotid and middle cerebral artery occlusion. Stroke 2007;38:2270-2274.

19 Fuentes B, Masjuan J, Alonso de Leciñana M, et al: Benefits of intravenous thrombolysis in acute ischemic stroke related to extra cranial internal carotid dissection. Dream or reality. Int J Stroke 2012;7:7-13.

20 Engelter ST, Dallongeville J, Kloss M, et al: Thrombolysis in cervical artery dissection data from the cervical artery dissection and ischaemic stroke patients (CADISP) database. Eur J Neurol 2012;19:1199-1206.

21 Arnold M, Nedeltchev K, Sturzenegger M, et al: Thrombolysis in patients with acute stroke caused by cervical artery dissection: analysis of 9 patients and review of the literature. Arch Neurol 2002;59:549-553.

22 Baumgartner RW, Georgiadis D, Nedeltchev $\mathrm{K}$, et al: Stent-assisted endovascular thrombolysis versus intravenous thrombolysis in internal carotid artery dissection with tandem internal carotid and middle cerebral artery occlusion. Stroke 2008;39:e27-e28.

23 Rudolf J, Neveling M, Grond M, Schmulling S, Stenzel C, Heiss WD: Stroke following internal carotid artery occlusion - a contra-indication for intravenous thrombolysis?. Eur J Neurol 1999;6:51-55.

24 Georgiadis D, Lanczik O, Schwab S, et al: IV thrombolysis in patients with acute stroke due to spontaneous carotid dissection. Neurology 2005;64:1612-1614.
25 Vergouwen MD, Beentjes PA, Nederkoorn PJ: Thrombolysis in patients with acute ischemic stroke due to arterial extracranial dissection. Eur J Neurol 2009;16:646-649.

26 Wahlgren N, Ahmed N, Dávalos A, et al: Thrombolysis with alteplase for acute ischaemic stroke in the safe implementation of thrombolysis in stroke-monitoring study (SITS-MOST): an observational study. Lancet 2007;369:275-282.

27 Ahmed N, Wahlgren N, Grond M, et al: Implementation and outcome of thrombolysis with alteplase 3-4.5 $\mathrm{h}$ after an acute stroke: an updated analysis from SITS-ISTR. Lancet Neurol 2010;9:866-874

28 Engelter ST, Traenka C, Von Hessling A, et al: Diagnosis and treatment of cervical artery dissection. Neurol Clin 2015;33:421-441.

29 Zinkstok SM, Vergouwen MD, Engelter ST, et al: Safety and functional outcome of thrombolysis in dissection-related ischemic stroke: a meta-analysis of individual patient data. Stroke 2011;42:2515-2520.

30 Engelter ST, Brandt T, Debette S, et al: Antiplatelets versus anticoagulation in cervical artery dissection. Stroke 2007;38:2605-2611.

31 Hacke W, Kaste M, Bluhmki E, et al: Thrombolysis with alteplase 3 to 4.5 hours after acute ischemic stroke. N Engl J Med 2008;359: 1317-1329.

32 Engelter ST, Rutgers MP, Hatz F, et al: Intravenous thrombolysis in stroke attributable to cervical artery dissection. Stroke 2009;40: 3772-3776.

33 Caplan LR: Dissections of brain-supplying arteries. Nat Clin Pract Neurol 2008;4:34-42.

34 Patel RR, Adam R, et al: Cervical carotid artery dissection: current review of diagnosis and treatment. Cardiol Rev 2012;20:145-152.

35 Tsivgoulis G, Zand R, Katsanos A, et al: Safety and outcomes of intravenous thrombolysis in dissection-related ischemic stroke: an international multicenter study and comprehensive meta-analysis of reported case series. J Neurol 2015;262:2135-2143.

36 Saeed AB, Shuaib A, Al-Sulaiti G, et al: Vertebral artery dissection: warning symptoms, clinical features and prognosis in 26 patients. Can J Neurol Sci 2000;27:292-296.

37 BudimkićMS,BerisavacI,Beslać-Bumbaširević $\mathrm{L}$, et al: Intravenous thrombolysis in the treatment of ischemic stroke due to spontaneous artery dissection. Neurologist 2012;18:273276. 